\title{
Vertical Bone Augmentation Using Three-dimensionally Printed Cap in the Rat Calvarial Partial Defect
}

\author{
JOONG-MIN KIM ${ }^{1,2^{*}}$, JOONG-HYUN KIM ${ }^{3,4 *}$, BYEONG-HAN LEE ${ }^{3}$ and SEOK HWA CHOI \\ ${ }^{1}$ e-Well Dental Hospital, Seoul, Republic of Korea; \\ ${ }^{2}$ Department of Dentistry, Graduate School of Medicine, Korea University, Seoul, Republic of Korea; \\ ${ }^{3}$ Laboratory Animal Center, Osong Medical Innovation Foundation, Cheongju, Republic of Korea; \\ ${ }^{4}$ Department of Nanobiomedical Science and BK21 PLUS NBM Global Research Center for Regenerative Medicine, \\ Dankook University, Cheonan, Republic of Korea; \\ ${ }^{5}$ Department of Veterinary Surgery, College of Veterinary Medicine, \\ Chungbuk National University, Cheongju, Republic of Korea
}

\begin{abstract}
Background/Aim: Lost alveolar bone is commonly restored by distraction osteogenesis or bone blocks for substantial vertical bone augmentation (VBA), that is applied in conjunction with a barrier system. This study was performed to determine whether volume control of a threedimensional (3D) printed nylon cap in the rat calvarial partial thickness bone defect would induce qualitative and quantitative differences in vertical bone regeneration. Materials and Methods: A rat calvarial partial thickness bone defect was prepared and the $3 D$ cap covered the defect to induce VBA, while the control group was left without cap placement. After six weeks the animals were sacrificed, and the calvaria were prepared for micro-CT $(\mu C T)$ and histology. Results: Quantitative $\mu C T$ results showed that our cap system has significant osteoconductive properties, and the histology slide revealed new bone filled inside the cap. Conclusion: The results clearly showed that this system was successful for $V B A$ in a research animal model.
\end{abstract}

Even while recent advances in biomaterials and implant techniques have contributed to increased use of dental implants, excessive bone resorption prevents the positioning of oral implants (1-3). Surgical augmentation techniques

This article is freely accessible online.

*These Authors contributed equally to this study.

Correspondence to: Seok Hwa Choi, DVM, Ph.D., Department of Veterinary Surgery, College of Veterinary Medicine, Chungbuk National University, Cheongju, Chungbuk, 28644, Republic of Korea. Tel: +82 432613144, Fax: +82 432613224, e-mail: shchoi@cbu.ac.kr

Key Words: Vertical bone augmentation, three-dimension, printing, rat calvarial partial defect. such as bone grafts, barrier membranes for guided bone regeneration (GBR), and distraction osteogenesis have been introduced for effective vertical bone augmentation (VBA) to achieve structural support for implantation (1-3). For bone regeneration in contour deficits in the craniofacial skeleton, various osteoconductive biomaterials have been developed and explored (1-3). As a promising option for tissue engineering applications, these biomaterials have been proposed for preclinical testing for VBA (4) with the GBR technique. GBR can be achieved by using a barrier that allows new bone to migrate into the space while preventing other cell types from interfering (5).

Animal models for studying tissue engineering are designed to present and enable conclusions by obtaining preclinical representatives, which is applicable to clinical translation (6). The small animal model of this study is designed for VBA using GBR by the following criteria: space maintenance by rigid immobilization to prevent micromotion of graft, exclusion of the cell and tissue infiltration from the surrounding environment, and clot stabilization (1-3). In rodents, the calvaria serves to test extracortical vertical bone formation as an experimental model of the jaw bone of human, due to their similar developmental pathway of bone formation $(7,8)$. To reduce required animal number, bilateral implantation was undertaken $(9,10)$.

The application of 3D printed products to bioprint replacements for lost tissue has attracted great attention. As printers and software become more widely available, 3D printing is being successfully used in medicine (11). By accumulating the printable biomaterials layer-by-layer, 3D printers manufacture clinically available patient-specific implants and devices. In addition to the aforementioned uses, new 3D printing techniques have been applied by biotechnology firms and academia to study tissue response for possible use in tissue engineering $(4,12)$. 
To accurately assess the use of osteoconductive graft to obtain vertical bone regeneration, effective in vivo research tools should be established. Although there have been many fundamental studies of 3D printing scaffold system for VBA, no trial has attempted to create a printed cap as a barrier system. Metal or polymer caps are most often made with a molding system, changing the dimensions (diameter $\mathrm{x}$ height) of the cap is not easy in this system (Figure 1). This research focused on development of a 3D printed cap using polymer filaments with a commercially available table top $3 \mathrm{D}$ printer. The aim of the present study was to introduce an easily manufactured, volume controllable 3D printed cap system for VBA in vivo study.

\section{Materials and Methods}

Manufacturing $3 D$ printed cap. The cap design (internal dimension: diameter $5.5 \mathrm{~mm}$ and height $2 \mathrm{~mm}$ ) was created using computer program (SketchUp, Trimble Navigation Limited, Sunnyvale, CA, USA), and fabricated using a commercial FDM 3D printer (NPMendel, Opencreators, Seoul, Korea) with medical grade nylon (Taulman 618 Nylon, Taulman 3D, LLC, St Louis, USA) (Figure 2). The printer head extruded polymer filament on the bed according to a designed pattern. The filament feeder bed is raised and the extruder laid down the nylon filament from the feeder bed and evenly laid nylon onto the deposition bed. The process is repeated, layer by layer, until the CAD image is completely printed. After complete printing, the caps were sterilized using EO gas, and kept clean until use.

Placement of the cap in a rat calvarial partial defect. Eleven-weekold male Sprague-Dawley (SD) rats (n=6) (Daehan Biolink Co., Ltd, Korea) were prepared for this study. The rats were acclimatized to the environment for seven days before use, kept in barrier condition with $12 \mathrm{hr}$ light-dark cycle of equal time and access to water and food ad libitum. The experimental procedure was approved by the Institutional Animal Care and Use Committee of the Dankook University. After preparing two partial defects, defects were allocated to a control group (without cap: w/o cap), and the study group (with cap: w/ cap). General anesthesia was obtained by intramuscular administration of ketamine hydrochloride $(80 \mathrm{mg} / \mathrm{kg})$ and xylazine hydrochloride $(10 \mathrm{mg} / \mathrm{kg})$. After shaving, the surgical site was prepared with iodine and $70 \%$ ethanol. A 15 millimeters mid-sagittal linear incision was made through the skin, and a fullthickness flap, including periosteum, was reflected to expose the calvarial bone. To create the partial defect on the exact position on the parietal bone, and to control the depth of the defect, two guide plates were positioned on each side and fixed using screws for the press-fit stabilization (Figure 3A). Two circular partial defects 5.5 $\mathrm{mm}$ in diameter and $0.5 \mathrm{~mm}$ depth were made on each side of the parietal bone with the use of a LS-Reamer (Neobiotech, Seoul, Korea) under irrigation with sterile saline (Figure 3B). After removing the guide plates (Figure $3 \mathrm{C}$ ), the 3D printed rigid polymer caps were stabilized in each animal (Figure 3D). The soft tissues and periosteum were sutured with absorbable sutures (4-0 Vicryl ${ }^{\circledR}$, Ethicon, Germany) for total coverage, and the skin was closed with non-absorbable suture material (4-0 Prolene ${ }^{\circledR}$, Ethicon, Germany). After the operation, the animals were evaluated daily throughout the experiment periods to check for possible dehiscence.

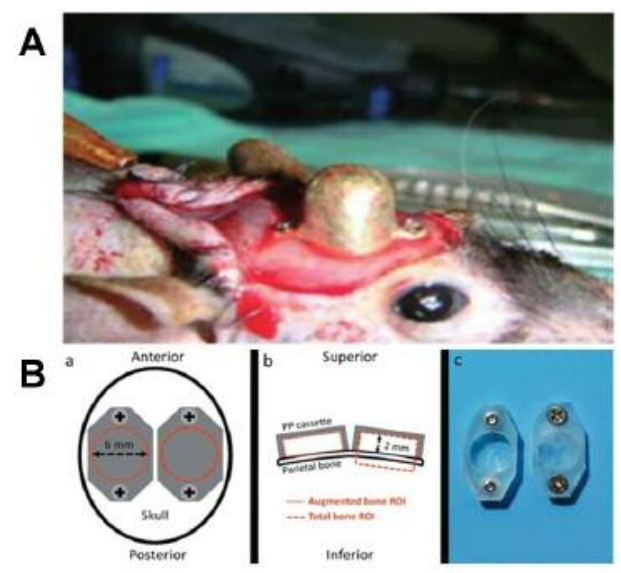

Figure 1. Earlier cap design for the VBA on the rat calvaria. (A) Gold dome fixed to the calvaria (from Ref. (17)). (B) Schematic representations of the polypropylene cassettes. (a) Top view showing implant positioning and (b) a coronal section through the center of the implants. (c) A digital photo showings bottom and top views of the polypropylene cassette s and stainless-steel screws (from Ref. (4)).

Microcomputed tomography. Six weeks after the operation, the animals were euthanized by $\mathrm{CO}_{2}$ inhalation, and the calvarial bones were harvested without removing the cap (Figure 4A). The samples were fixed in $4 \%$ neutralized buffered formalin (NBF) for microcomputed tomographic evaluation and histology (Figure 4B). After removing screws from the cap, the samples were scanned using a Skyscan 1176 In Vivo micro-CT system (Bruker microCT, Kontich, Belgium). Scans were performed using $65 \mathrm{kVp}, 378 \mu \mathrm{A}, 200 \mathrm{~ms}$ exposure, with $\mathrm{Al} 1 \mathrm{~mm}$ filter, and resulted in images with $17.93 \mu \mathrm{m}$ image pixel size. Volume of interest (VOI) is defined as a space occupying all the space inside the cap by the experimental group. In the control group, the VOI of the experimental group was taken from the experimental group and the same space was examined. The $\mu \mathrm{CT}$ $3 \mathrm{D}$ images were reconstructed (Figure 4C) and the percentage new bone volume, bone surface, and bone surface density were analyzed by a $\mu \mathrm{CT}$ program, using CTAn (Bruker microCT) (Figure 4D).

Histological observation. After decalcification using RapidCalTM solution (BBC Chemical Co., Stanwood, WA, USA), the samples were dehydrated in an ascending ethanol series (from $70 \%$ to $100 \%$ ). The cap was removed after paraffin infiltration (Figure 4E), and the samples were bisected and embedded. The blocks were sectioned $(5 \mu \mathrm{m})$ and stained with hematoxylin and eosin (H\&E) from the center plane of the sample (Figure 4F). The images were taken using light microscope (IX71, Olympus, Tokyo, Japan).

Statistical analysis. All data are presented as mean \pm standard deviation. Differences between groups were assessed by paired $t$-test. Null hypotheses of no difference were rejected if $p$-values were less than 0.05 .

\section{Results}

Application of $3 D$ printed cap. Rigid, nonporous hollow caps and guide plates were manufactured using a 3D printer. The cap was cylindrical, and the guide plate was designed based 

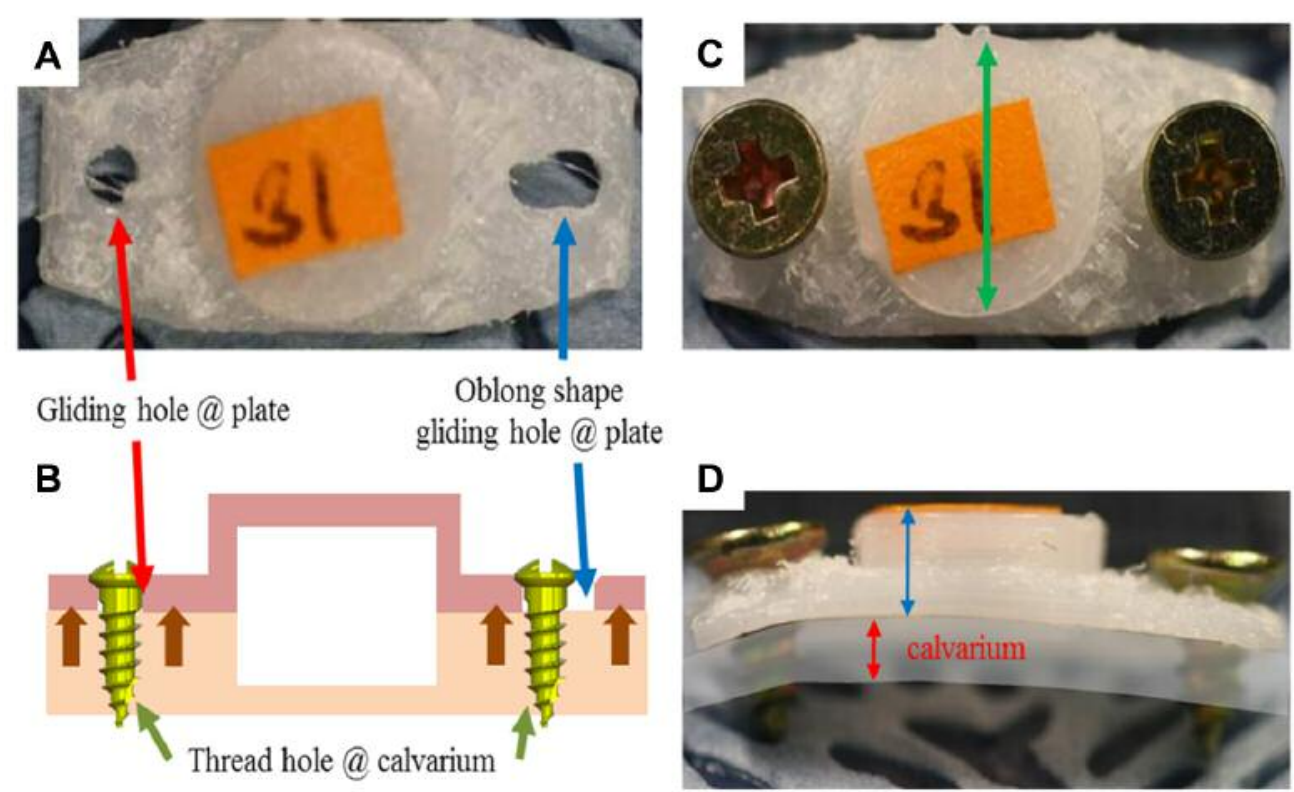

Figure 2. Custom-made 3D printed cap for the VBA on the rat calvaria. (A) Gliding hole was applied to this design. (B) Schematic image for the relation between the cap and calvaria. The screw inserted as a lag screw, with a gliding hole in the cap (red arrow), and a thread hole in the calvaria (blue arrow) and compression force (brown arrow) is applied between cap and calvaria for complete occlusion. (C) Top view of the cap with screw. Doubleheaded green arrow shows the diameter of the cap. (D) Side view of the cap with screw. White blurry area with double-headed red arrow indicates rat calvarial thickness. Double-headed blue arrow indicates cap height. Height and diameter of the cap can be easily modified by computer program.
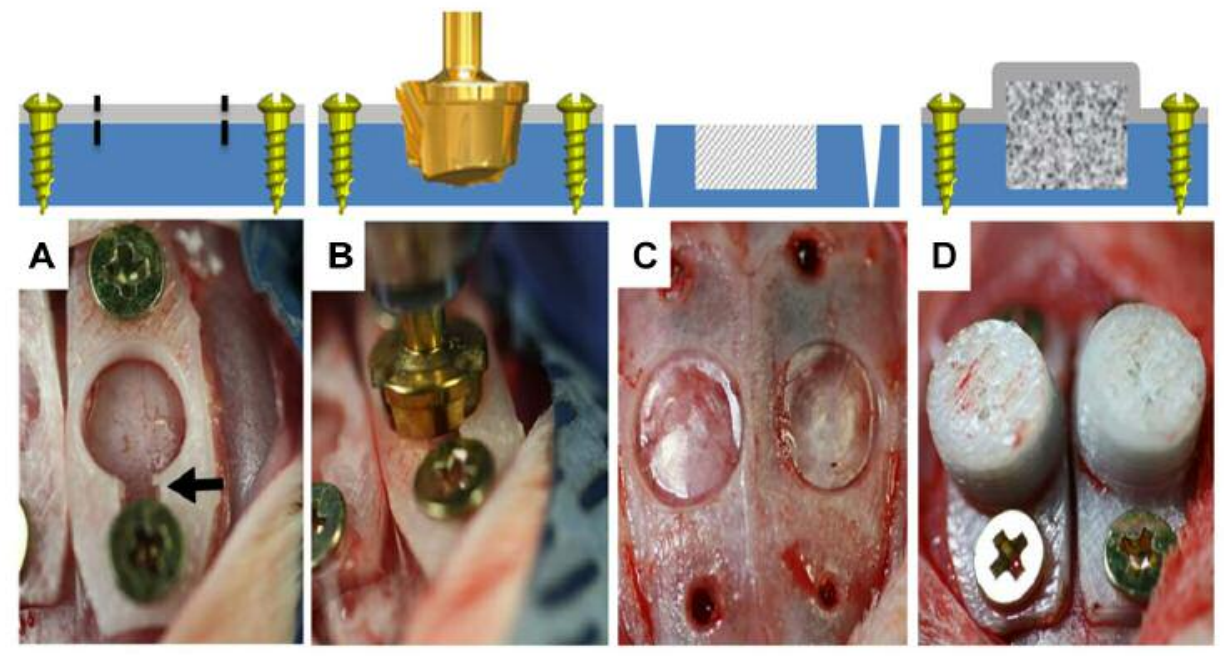

Figure 3. Experimental scheme and procedure of VBA in a rat calvarial partial bone defect and stabilization of the 3D printed cap. (A) Press-fit stabilization of the custom-made guide-plate on the rat calvaria and initial insertion of the screws. The gap between screw and center hole (black arrow) is the entrance for the irrigation with sterile saline to cool-down the bone during the grinding process. (B) Bone bed preparation by grinding bone using LS-reamer. The depth of the partial bone defect can be adjusted by changing thickness of the guide plate. (C) Flat-bottomed bone bed, after removing the guide plate. (D) Stabilization of the custom-made 3D printed polymer cap by fitting the retention screws (modified from Ref (20)).

on the cap dimensions. After printing, caps and guide plates were sterilized using EO gas, and kept until use. After surgery, the soft tissues for all animals healed uneventfully for six weeks. No abscess or soft tissue dehiscence were observed at the skin suture site. After assessing clinical findings, image analysis and histological observations for the cap implanted site were performed.

Macroscopic tissue responses within the $3 D$ printed cap. Clinical macroscopic view following removal of the cap 


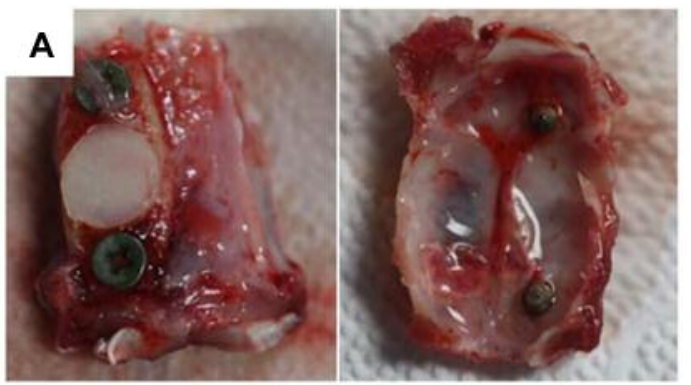

Harvesting samples after sacrifice

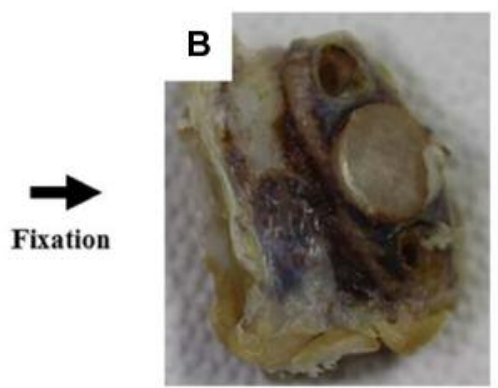

$1 \mu \mathrm{CT}$ imaging
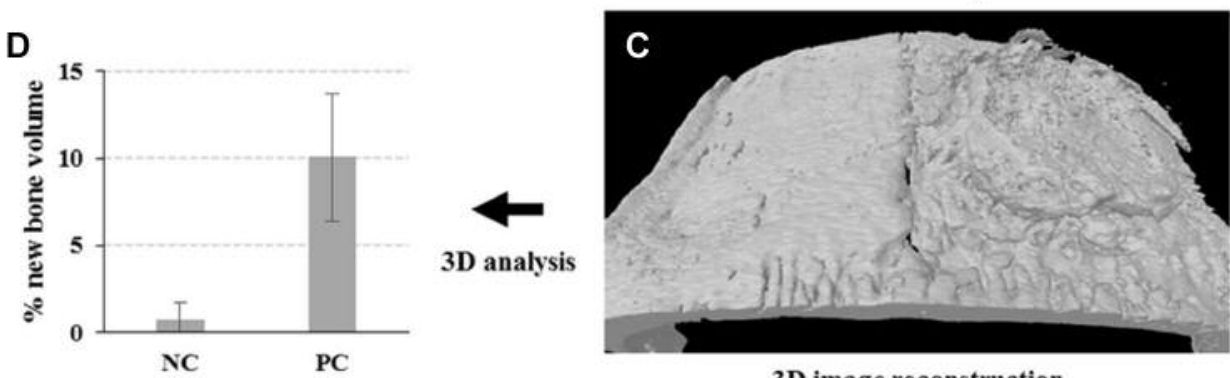

3D image reconstruction

Remove cap

E

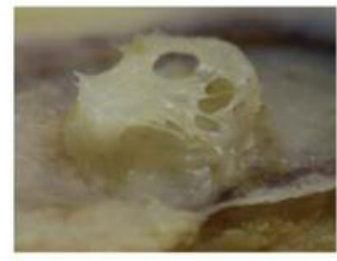

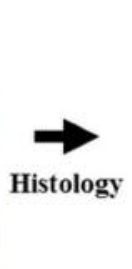

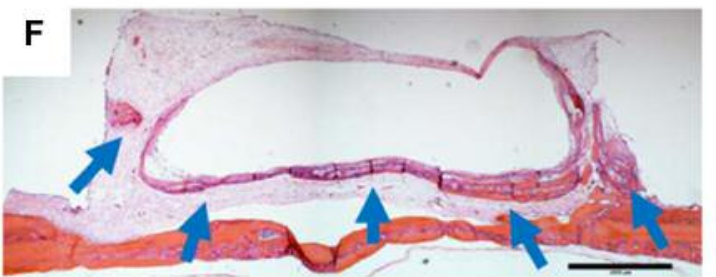

Figure 4. Micro CT analysis and histological findings at 6 weeks after operation. (A) Calvarial bone and the cap were harvested from rats. (B) The samples were fixed for $24 \mathrm{~h}$. (C) After fixation, $\mu \mathrm{CT}$ images were taken for the $3 D$ image reconstruction and analysis. (D) $3 D$ analysis for new bone formation. (E) After removing cap, newly formed tissues were seen on the rat calvarial partial thickness bone defects. (F) Longitudinal sections of the VBA area within cap. Histology showed the new tissue extended vertically from the rat calvaria and occupied the space under the cap. Arrow points the regenerated bone.

showed that most space inside the cap remained empty with irregular new tissue formed. This vertically-augmented tissue appeared non-homogenous, with soft yellowish features only at the lateral borders in contact with inside surface of the cap. In the control group, it was difficult to see the newly generated tissue on the defect surface.

Micro-computed tomographic results. All of the quantitative measurements were taken along the six samples from each group, Figure 5A reports the percentages (\%) of the newly formed bone volume within the cap area and particulate specimens. The cap specimens had a mean $\%$ of $10.06 \pm 3.63$ and the control showed a mean $\%$ of $0.74 \pm 0.96(p<0.0007)$. Figure 5B reports the surface of new bone fill in the area within the cap $\left(\mathrm{mm}^{2}\right)$. The mean values reported were
$96.71 \pm 33.26$ for the cap group and $11.63 \pm 15.09$ for the control $(p<0.002)$. Figure 5C reports the surface bone density $(1 / \mathrm{mm})$. The mean values reported were $1.43 \pm 0.42$ for the cap group and $0.18 \pm 0.23$ for the control $(p<0.0009)$. Representative 3D reconstructed $\mu \mathrm{CT}$ images of the VBA induced bone without cap and with cap (Figure 5D).

In vivo biocompatibility and histological findings. Overview images of the H\&E stained longitudinal sections are shown in Figure 5E. The histological examination showed that our cap system did not induce inflammatory response in an in vivo condition. Newly-generated tissue was not infiltrated with erythrocytes and inflammatory cells, and grew along the internal surface of the cap. This tissue was mostly composed of connective tissue which encapsulated newly-formed bone 

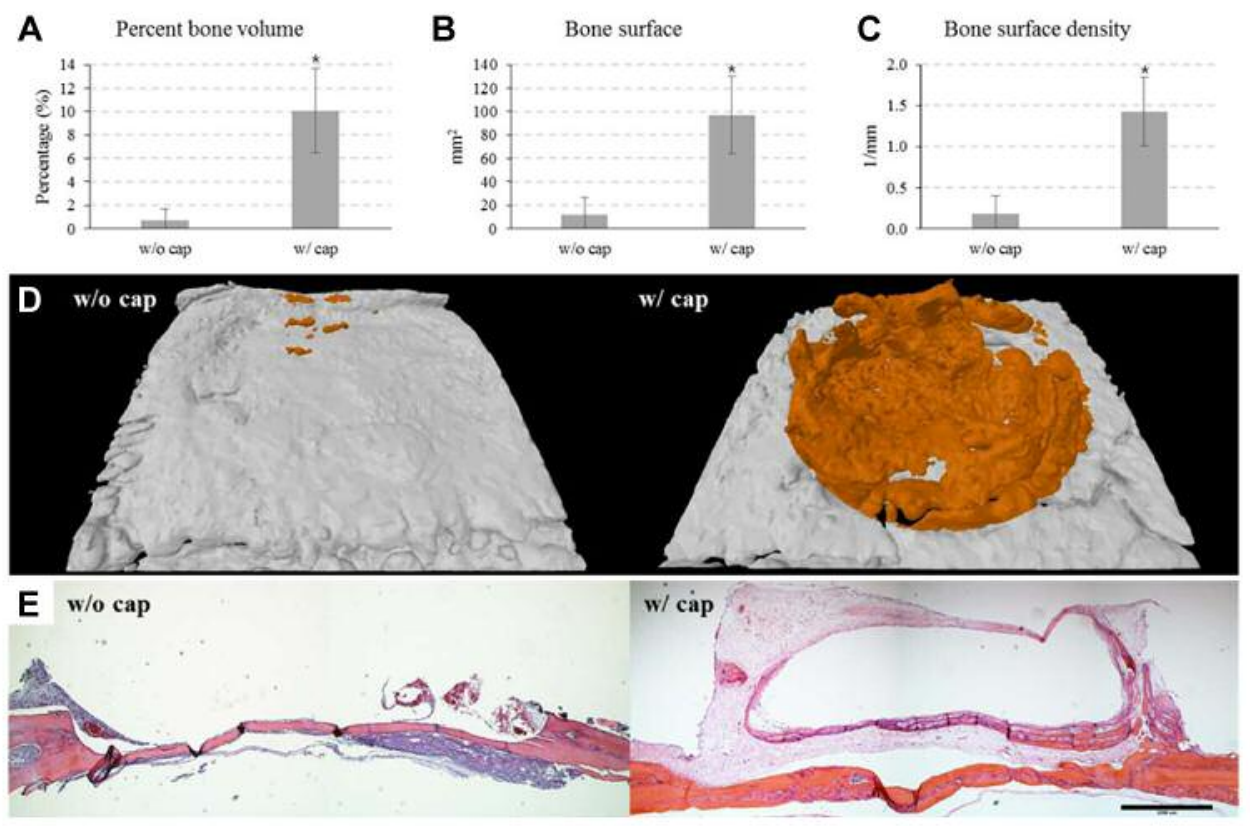

Figure 5. Bone induction effect of the VBA without cap (w/o cap) and with cap (w/ cap) in the rat calvarial partial defect. (A-C) Graphs show the mean \pm standard deviation of percentage new bone volume, bone surface, and bone surface density in the two groups $(n=6)$. *Statistically significant difference between groups (student-t test; $p<0.05$ ). (D) Representative $3 D$ reconstructed $\mu C T$ images of the VBA induced bone without cap and with cap. (E) H\&E stained longitudinal sections showed that our cap system did not induce inflammatory response in in vivo condition. Magnification $\times 40$, scale bar $=1000 \mu \mathrm{m}$.

tissue. New bone appeared mature and continuous from the host calvarial bone, whereas there was almost no new bone formed on the defect surface when the cap was not applied.

\section{Discussion}

The present study assessed the macroscopic, $\mu \mathrm{CT}$ and histological outcomes of a 3D printed cap system implanted bilaterally on rat calvarial partial defect in vertical bone augmentation. Rigid immobilization of the sample and isolation by close occlusion from the surrounding environment were evaluated. The results clearly showed that this technique was successful for VBA research to assess osteoconductivity. Six animals were enrolled and completed the study for a total of 12 sites. Despite of low number of rats in each group, in vivo results showed minimal intragroup variation, and this sample size can be considered sufficient as a proof of this study.

Three-dimensional printing, a desktop fabrication or additive manufacturing system, is a process whereby an object is printed layer by layer. The computer-created 3D design file is sent to the printer, which prints the design on the printing plate layer-by-layer to produce the desired $3 \mathrm{D}$ structure (12). A widely used method to produce 3D printed items is fused deposition modeling (FDM) (13). FDM produces $3 \mathrm{D}$ printed items from thermoplastic filaments with a simple heating process to extrude the materials. The advantage of FDM 3D printers is that it is cost-competitive with other 3D printing machines, and this is why we chose the FDM 3D printer to fabricate 3D printed cap for this study. Professional printers for fabricating biomaterials cost more (11), but the NP-Mendel is a cheap desktop 3D printer that can use several polymeric filament materials. Depending on the printing materials, this printer can create products with a range of textures, colors, strength, and malleability. In addition to the preparation of the hardware, software for $3 \mathrm{D}$ modelling was required. These programs are likewise expensive (12), but there are a number of free 3D printing programs, which enables users to transform objects from a projection on a monitor to a 3D-printed organ or tissue (11). SketchUp is one of the most popular programs, which allows anyone to create $3 \mathrm{D}$ print templates.

Thermoplastics, which have a low melting temperature, are considered advanced technology biomaterials to create highquality parts for medical applications, and have been applied to replace mold fabricated polymers and metals. A variety of types of thermoplastics (Acrylonitrile butadiene styrene (ABS), polylactic acid (PLA), and nylon filaments) are commonly used printable materials for commercial 3D printer using FDM processes. Nylon is the first nylon co-polymer specifically formulated for $3 \mathrm{D}$ printing. Compared with ABS 
and PLA, nylon is expensive, and is not US Food and Drug Administration (FDA) approved material. However, 618 nylon has high strength, pliability, chemical resistance and high thermal durability (14). These properties make 3D printing using nylon filament no harder than printing with any other material. A good quality nylon material provides good layer adhesion, and results in a fault-free and small intricate product. Nylon products show very nice finish quality, with a smooth silky finish. Furthermore, the products are reproducible. Although Taulman 680 nylon is currently FDA approved, the 618 nylon used in this study was not approved yet. However, 618 nylon is inert to the body and contains no organic compounds. Because it is indigestible, 618 nylon passes through the system and is biocompatible (14).

To study in vivo the vertical bone regenerative property of the osteoconductive materials under GBR conditions, it is important to provide space to maintain the initial geometry of each scaffold. The scaffold needs to be fixed tight to achieve rigid immobilization and to limit diffusion or tissue infiltration from the overlying environment. A barrier system is commonly applied to achieve GBR condition, and this system should have suitable occlusive properties, maintain space for vertical bone regeneration, and be biocompatible (5). The barrier system we developed in this study used rigid, nonporous hollow caps, printed by commercial 3D printer with commercial material. This has not been reported in any preclinical animal model experiments.

The results from the $\mu \mathrm{CT}$ image analysis demonstrated significant osteoconduction when the partial defects were covered by the $3 \mathrm{D}$ printed rigid cap system. The cap system showed statistically higher bone volume in the areas within the cap compared with the control, and higher bone surface and bone surface density of regenerated bone within the area of volume of interest (VOI). These findings suggest that our cap system maintained the inner space without interference with outer tissue invasion, and significantly induced vertical bone regeneration.

Some researchers have reported that when applying particulate grafts for the VBA procedures, dimensions of a bone block graft needed to be changed on each case (15). To keep the graft undisturbed during a vertical bone regeneration, a space maintaining barrier (membrane, mesh, or the like) seems to be necessary (16). Our study design kept these needs for VBA principles. Our in vivo design can be applied to evaluate the osteoconductive effect on the graft placed in the cap without interference from the outer microenvironment. The new bone had clear integration with the basal host bone at the interface level near the cap, but the basal host bone and the newly formed bone showed a separation at the center part. However, this result may show that our cap system has tight occlusion. Fibrous tissues did not grow into the cap, and the new bone growing on the inner surface of the cap was not disturbed. Non-degradable nylon was used, to prevent the effect of the degraded material debris on VBA. Sufficient occlusion of the cap and the structural integrity during the study period may contribute to the predictable extracortical bone formation (17). Cortical perforations on bone formation under rigid domes accelerated bone formation in preclinical trials (18, 19). In this study, cortical perforation was not performed, but the animal host bone had enough VBA.

Our system was applied not only to the osteoconductive study, but also the osseointegration study. Some researchers studied surface modification by chemical functionalization of titanium (Ti) by covalently anchoring a synthetic doublebranched molecule (20). This modification stimulated differentiation of human mesenchymal stem cells into the osteoblastic lineage, and was efficient in promoting new bone growth in vivo in a rat calvarial defect. Custom-made rod-type Ti implants (5.5 $\mathrm{mm}$ diameter, $5 \mathrm{~mm}$ long) were covered by $3 \mathrm{D}$ printed rigid polymer caps, and the cap and Ti constructs were secured to the calvarial bone using fixation screws via its anchoring rings. After two and four weeks, the animals were sacrificed and the samples were prepared for histology. In this study, the cap height modified suitable for long sample size, and the animal showed a local necrosis on the skin overlying the cap by gross observation. However, the wound from the necrosis resulted in scab formation, and the histological results revealed that these focal responses did not affect the study results. Our cap system clearly showed Ti surface modification capacity to the osseointegration in a rat calvarial partial defect model.

The findings of our study encourage a new VBA in vivo study design for an ideal bone substitute in the variable form of a bone graft for vertical bone regeneration. The 3D printed rigid cap resulted in proper VBA without complications in the calvaria of rats.

\section{Data Availability}

Previously reported figure data were used to support this study and are available at $10.1111 / \mathrm{j} .1708-8208.2012 .00452 . x$, and 10.1002/jbm.a.34878. These prior studies are cited at relevant places within the text as references (6) and (7).

\section{Acknowledgements}

This research was supported by Basic Science Research Program through the National Research Foundation of Korea (NRF) funded by the Ministry of Education (2015R1D1A1A01060583 and 2016R1A6A3A11935091), Republic of Korea.

\section{References}

1 Slotte C and Lundgren D: Augmentation of calvarial tissue using non-permeable silicone domes and bovine bone mineral. An experimental study in the rat. Clin Oral Implants Res 10(6): 468476, 1999. 
2 Klijn RJ, van den Beucken JJ, Felix Lanao RP, Veldhuis G, Leeuwenburgh SC, Wolke JG, Meijer GJ and Jansen JA: Three different strategies to obtain porous calcium phosphate cements: Comparison of performance in a rat skull bone augmentation model. Tissue Eng Part A 18(11-12): 1171-1182, 2012.

3 Polimeni G, Albandar JM and Wikesjo UM: Prognostic factors for alveolar regeneration: Effect of space provision. J Clin Periodontol 32(9): 951-954, 2005.

4 Kinard LA, Dahlin RL, Henslee AM, Spicer PP, Chu CY, Tabata Y, van den Beucken JJ, Jansen JA, Young S, Wong ME, Kasper FK and Mikos AG: Tissue response to composite hydrogels for vertical bone augmentation in the rat. J Biomed Mater Res A 102(7): 2079-2088, 2014.

5 Anderud J, Jimbo R, Abrahamsson P, Isaksson SG, Adolfsson E, Malmstrom J, Kozai Y, Hallmer F and Wennerberg A: Guided bone augmentation using a ceramic space-maintaining device. Oral Surg Oral Med Oral Pathol Oral Radiol 118(5): 532-538, 2014.

6 Gruskin E, Doll BA, Futrell FW, Schmitz JP and Hollinger JO: Demineralized bone matrix in bone repair: History and use. Adv Drug Deliv Rev 64(12): 1063-1077, 2012.

7 Lundgren D, Lundgren AK, Sennerby L and Nyman S: Augmentation of intramembraneous bone beyond the skeletal envelope using an occlusive titanium barrier. An experimental study in the rabbit. Clin Oral Implants Res 6(2): 67-72, 1995.

8 Verna C, Dalstra M, Wikesjo UM and Trombelli L: Healing patterns in calvarial bone defects following guided bone regeneration in rats. A micro-ct scan analysis. J Clin Periodontol 29(9): 865-870, 2002.

9 Matsui A, Anada T, Masuda T, Honda Y, Miyatake N, Kawai T, Kamakura S, Echigo S and Suzuki O: Mechanical stress-related calvaria bone augmentation by onlayed octacalcium phosphatecollagen implant. Tissue Eng Part A 16(1): 139-151, 2010.

10 Martinez-Sanz E, Ossipov DA, Hilborn J, Larsson S, Jonsson $\mathrm{KB}$ and Varghese OP: Bone reservoir: Injectable hyaluronic acid hydrogel for minimal invasive bone augmentation. J Control Release 152(2): 232-240, 2011.

11 Murphy SV and Atala A: 3d bioprinting of tissues and organs. Nat Biotechnol 32(8): 773-785, 2014.

12 Soliman Y, Feibus AH and Baum N: 3d printing and its urologic applications. Rev in Urol 17(1): 20-24, 2015.
13 Hwang S, Reyes EI, Moon K-s, Rumpf RC and Kim NS: Thermo-mechanical characterization of metal/polymer composite filaments and printing parameter study for fused deposition modeling in the $3 \mathrm{~d}$ printing process. J Electron Mater 44(3): 771-777, 2015.

14 Amanov E, Nguyen TD and Burgner-Kahrs J: Additive manufacturing of patient-specific tubular continuum manipulators. Proc of SPIE 9415: 9415P-6, 2015.

15 Cordaro L, Amade DS and Cordaro M: Clinical results of alveolar ridge augmentation with mandibular block bone grafts in partially edentulous patients prior to implant placement. Clin Oral Implants Res 13(1): 103-111, 2002.

16 Rocchietta I, Simion M, Hoffmann M, Trisciuoglio D, Benigni $\mathrm{M}$ and Dahlin C: Vertical bone augmentation with an autogenous block or particles in combination with guided bone regeneration: A clinical and histological preliminary study in humans. Clin Implant Dent Relat Res 18(1): 19-29, 2016.

17 Zigdon H, Lewinson D, Bick T and Machtei EE: Vertical bone augmentation using different osteoconductive scaffolds combined with barrier domes in the rat calvarium. Clin Implant Dent Relat Res 16(1): 138-144, 2014.

18 Min S, Sato S, Murai M, Okuno K, Fujisaki Y, Yamada Y and Ito $\mathrm{K}$ : Effects of marrow penetration on bone augmentation within a titanium cap in rabbit calvarium. J Periodontol 78(10): 1978-1984, 2007.

19 Majzoub Z, Berengo M, Giardino R, Aldini NN and Cordioli G: Role of intramarrow penetration in osseous repair: A pilot study in the rabbit calvaria. J Periodontol 70(12): 1501-1510, 1999.

20 Fraioli R, Dashnyam K, Kim JH, Perez RA, Kim HW, Gil J, Ginebra MP, Manero JM and Mas-Moruno C: Surface guidance of stem cell behavior: Chemically tailored co-presentation of integrin-binding peptides stimulates osteogenic differentiation in vitro and bone formation in vivo. Acta Biomater 43: 269-281, 2016.
Received May 9, 2018

Revised June 14, 2018

Accepted June 15, 2018 